\title{
Actualisation, risque et cacao. Les insuffisances de la théorie
}

Mme Véronique Alary, M. Jean-Marc Boussard

\section{Citer ce document / Cite this document :}

Alary Véronique, Boussard Jean-Marc. Actualisation, risque et cacao. Les insuffisances de la théorie. In: Économie rurale. N²59, 2000. pp. 64-74;

doi : https://doi.org/10.3406/ecoru.2000.5207

https://www.persee.fr/doc/ecoru_0013-0559_2000_num_259_1_5207

Fichier pdf généré le 09/05/2018 


\title{
Résumé
}

Lorsqu'on applique aux taux d'actualisation les valeurs effectivement prises par les prix et les taux d'intérêts courants, il est rare de trouver une "valeur actuelle» positive pour un investissement forestier. Dans les pays en développement qui connaissant généralement des taux d'intérêt élevés, l'application de la théorie de l'actualisation conduirait à l'arrachage de milliers d'hectares de plantation. Néanmoins, on observe que les gens continuent à planter des arbres. Ceci conduit à supposer que c'est le risque plus que la préférence pour le présent qui conditionne les comportements. Cette idée est illustrée par les résultats d'une enquête et d'un modèle sur auprès des planteurs de cacao du Cameroun, qui montrent que les prédictions de la théorie de l'actualisation ne correspondent pas au comportement réel des planteurs. On verra alors, d'une part que la théorie pure de l'actualisation devient sans objet, et que, d'autre part, il est possible de définir le taux d'actualisation de manière endogène.

\begin{abstract}
Discounting, risk and cocoa: theoretical failures - Given observed prices, current rates of discount seldom produce positive present values for forest investments. Especially in LDC's, where rates of interest are high, a «rational» investor should remove trees, and plant annual crops. Yet, it is not unusual to find tree planting farmers. In order to explain such behavior, one is forced to admit that the preference to present against futures consumption is less important than assumed by current theory, and should perhaps be replaced by dynamic risk aversion. These remarks are illustrated by a survey and a multi-period model of Cameroon cocoa planters. It is shown that the predictions of current theory are not conformable with observed facts. On the other hand, by endogenously defining the rate of discount from risk consideration, a better fit to reality is obtained.
\end{abstract}




\section{A ctualisation, risque et cacao Les insuffisances de la théorie}

Le cacaoyer est un arbre dont la durée de vie est de l'ordre du demi-siècle. Il réclame tous les ans des soins, dont l'intensité dépend de la volonté du planteur. Celui-ci peut s'en désintéresser et il obtient alors une production faible ou nulle, variable selon l'âge de l'arbre. Il peut, au contraire, y investir son temps, et, plus encore, son argent, s'il en a, sous forme d'engrais, de pesticides ou de plants hybrides. Les rendements s'accroissent alors de façon significative, dans des proportions connues des agronomes (ils disposent de tables qui sont de véritables fonctions de production technique et ces tables sont connues des agriculteurs, au moins pour leurs caractéristiques majeures), suivant toujours l'âge de la plantation. On est donc en principe dans les conditions standards pour appliquer la théorie économique classique de l'actualisation à la plantation de cacao, comme l'avait tenté Bateman (1965)'.

On peut cependant s'interroger sur la pertinence de ce critère, notamment quand on l'utilise dans des conditions «extrêmes", comme c'est le cas en foresterie. En effet, le taux d'actualisation utilisé pour pondérer les événements dans le temps est traditionnellement le taux d'intérêt sur le marché des capitaux, considéré comme le prix d'équilibre

1. Il est du reste remarquable que cette théorie ait été, à l'origine, le fait de forestiers, précisément confrontés à ce problème. Le critère de Faustmann (Faustmann, 1849) qui consiste à choisir les rotations forestières sur la base de la maximisation de la valeur actuelle d'une rotation renouvelée à l'infini est sans doute l'une des premières manifestations de la théorie de l'actualisation (Samuelson, 1995; Terreau, 1990). entre l'offre et la demande d'épargne, résultant de la confrontation de l'ensemble des préférences individuelles des opérateurs sur ce marché. Lorsqu'on applique aux taux d'actualisation les valeurs effectivement prises par les taux d'intérêt courants, il est rare de trouver une "valeur actuelle» positive pour les prix du bois observés et les coefficients techniques que l'on trouve en foresterie. Dans les pays en développement, qui connaissent généralement des taux d'intérêt élevés, l'application de la théorie de l'actualisation conduirait à l'arrachage de milliers d'hectares de plantation, et, plus généralement, à l'abandon de toute activité forestière.

Heureusement pour les croqueurs de chocolat (et sans doute aussi pas mal d'écologistes), ce n'est pas le cas: on trouve des gens pour planter du cacao et pour se préoccuper de la forêt tropicale. Ces êtres sont-ils irrationnels? Représentent-ils des formes primitives d'humanité, auxquelles il est urgent d'apprendre des rudiments d'économie, pour les faire entrer dans l'ère moderne du développement? Ou sont-ils au contraire extrêmement avisés, et largement en avance sinon dans la conscience théorique, du moins dans la pratique concrète de la science économique, sur la vaste majorité de nos contemporains?

Si la seconde interprétation est la bonne, l'étude du fonctionnement concret des planteurs de cacao devrait faire progresser notre compréhension de la théoric du capital ct dc l'investissement. C'est l'objet de cet article. Nous commencerons par essayer de décrire correctement le problème qui se pose au 
planteur - en s'appuyant sur une étude de terrain menée en 1993 et 1994 au Came- roun -, puis on montrera les implications de cette situation pour la théorie économique.

\section{Cas d'étude empirique: le cacao comme "assurance-vie» au Cameroun}

\section{Les insuffisances de la théorie de l'actualisation}

Une première remarque est que les planteurs de cacao ne plantent pas que du cacao: ils pratiquent aussi des cultures saisonnières ou annuelles, dites «vivrières», dont ils autoconsomment une partie et vendent le reste. Chaque année, ils doivent donc décider la part des ressources (ressources humaines et financières) qu'ils allouent à l'entretien des cacaoyères ou à la réalisation de cultures vivrières. Dans la mesure où les cultures vivrières sont aussi bien connues que les pratiques réalisables sur les cacaoyères, le problème est à peine compliqué par cette circonstance. Il est vrai que le nombre des différentes «rotations» possibles devient alors très grand. Mais cette difficulté est assez facile à surmonter en utilisant les moyens de la programmation linéaire multipériodique, dont on verra qu'elle permet, en pratique, de chercher la rotation optimale en maximisant la somme des consommations actualisées sur une durée de temps infinie (Leplaideur $e t$ al., 1971; Boussard, 1972).

Le problème n'est donc pas là. Deux circonstances donnent à la question de la gestion de la cacaoculture camerounaise une physionomie toute différente de celle de l'histoire néoclassique racontée jusqu'ici:

1. Le choix des cultures et des pratiques associées se situe dans un contexte de prix d'une extrême instabilité. Jusqu'à une date récente au Cameroun, le prix du cacao était soutenu, à un niveau toujours plus faible que celui du marché mondial et sans lien avec l'évolution des prix des autres biens, mais il était garanti; tandis que les prix des cultures vivrières flottaient au grès de marchés extrêmement instables. Depuis 1989-90, les prix du cacao ne sont plus soutenus: ceci est dû à l'effondrement des prix sur le marché international depuis 1985-86 et, aussi, à l'impossibilité de l'État camerounais de reverser les sommes accumulées dans la Caisse de stabilisation depuis sa création ${ }^{2}$. La dévaluation de $100 \%$ du franc CFA en 1994 et l'amélioration des conditions sur le marché international ont fait remonter le prix payé aux planteurs dans des proportions significatives, mais la dévaluation a aussi accru le prix des pesticides et des engrais dans des proportions similaires.

2. Les planteurs camerounais, et plus généralement africains, ne bénéficient pas de droits privatifs sur leur terre mais un droit d'usufruit (donc temporaire) et ne peuvent compter sur le cadastre ou des notaires ruraux impartiaux pour régler les multiples conflits fonciers d'ordre coutumier. Dans ce contexte, seules les cultures pérennes, comme le cacaoyer, ont permis de faire passer le droit d'usufruit de l'année à plusieurs décennies. De nos jours, le cacaoyer constitue toujours un bon «marqueur foncier», en délimitant la propriété des planteurs et de leurs descendants.

Tant l'instabilité des prix que l'absence de sécurité foncière confèrent au problème du planteur de cacao un caractère singulier, au point que la théorie de l'actualisation telle qu'elle vient d'être décrite - devient insuffisante pour rendre compte de son comportement. Il convient alors de formuler

2. Or, les années où les cours mondiaux étaient supérieurs au prix garanti (1976-1979 et 1984-1986), la Caisse de stabilisation des matières premières (ONCPB) au Cameroun a pu prélever annuellement en moyenne 60 milliards de F CFA, et jusqu'à 66 milliards de F CFA en 1984-1985 (G. Courade et al., 1991). Près de 220 milliards de F CFA auraient été accumulés durant la période 1979 à 1989. 
le problème autrement et dans des conditions qui conduisent à définir un taux d'actualisation reflétant la préférence des producteurs dans le temps, donc un taux d'actualisation en quelque sorte endogène, en tout cas spécifique à chaque planteur et qui, en vérité, ne mérite pas le nom de taux d'actualisation.

Graphique 1. Évolution des prix d'achat au producteur en francs courants et francs constants (base 1993) et des prix mondiaux du cacao de 1960 à 1994. F CFA $/ \mathrm{kg}$

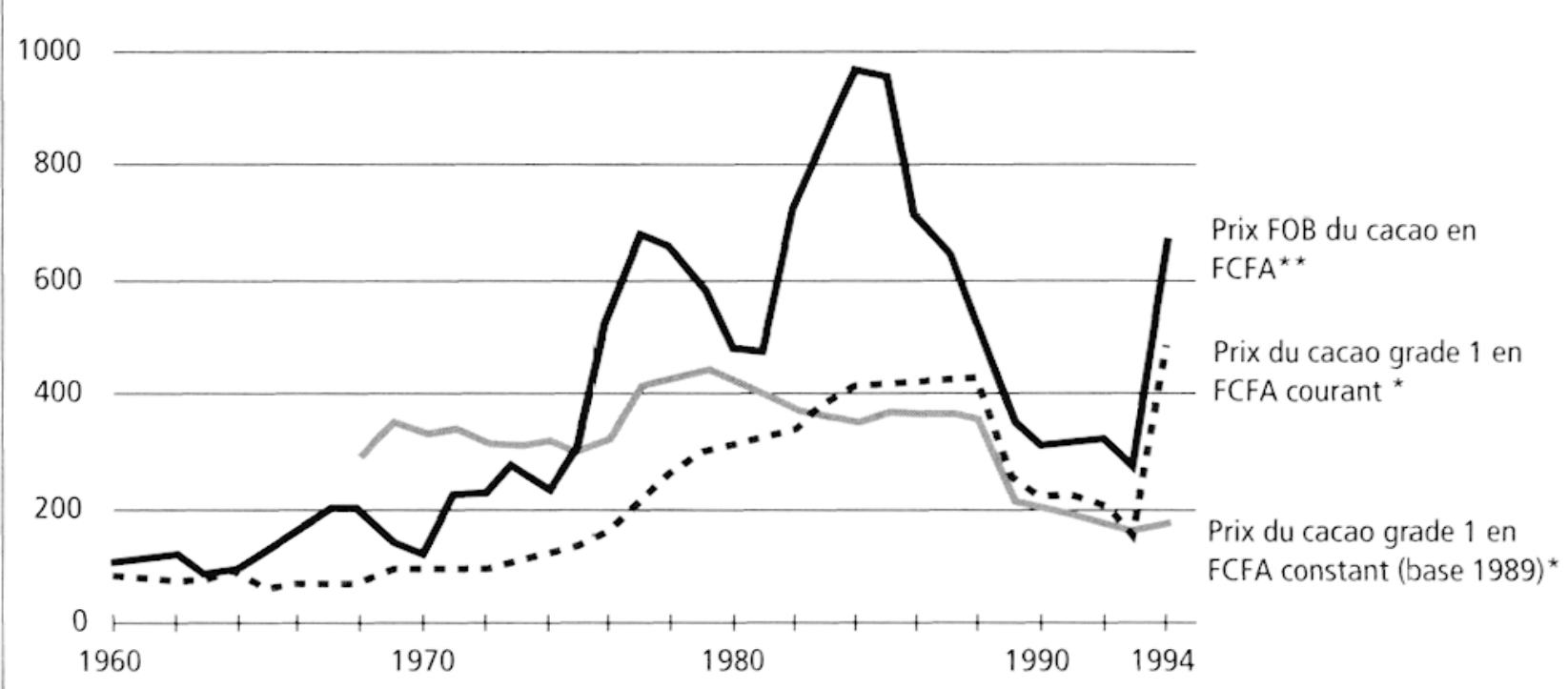

Source: *Prix d'achat aux planteurs: données ONCC/prix d'achat en francs constants calculés à partir de l'indice général des $p r i x$ (DSCN)

* Prix FOB en franc CFA courant: données ONCC

\section{Reformulation expérimentale du taux d'actualisation}

Pour étudier le processus de décision des producteurs camerounais et apprécier leurs intérêts dans la plantation, nous avons choisi une approche globale des systèmes de production mis en place à l'aide d'un système d'enquêtes à passages répétés. Deux enquêtes annuelles sur un échantillon permanent de 146 unités ont été réalisées, la première en 1993 (prix minimum garanti du cacao de $150 \mathrm{~F} \mathrm{CFA} / \mathrm{kg}$ ) et la deuxième en 1994 juste après la libéralisation des prix du cacao au producteur et la dévaluation du franc CFA. Notre étude s'est située dans quatre provinces (sept départements) de la zone cacaoyère qui assuraient en 1990 près de $75 \%^{3}$ de la production nationale de cacao.

3. D'après les données relevées dans le dernier recensement agricole de la production de cacao, réalisé par la CFA (Société des coopératives pour le développement rural) en 1986-1987.
L'analyse des réponses obtenues montre que, plus que des considérations de «coût d'opportunité» du capital, ou de préférence pour le présent, les attitudes face au risque façonnent le comportement des planteurs.

Tout d'abord, et quel que soit le prix du cacao, voire pour un cours très bas du cacao (150 F CFA/kg en 1993), peu de planteurs sont près à arracher les pieds de cacao, de peur de remettre en cause la propriété foncière et donc de compromettre le devenir des descendants. A la question posée en 1993: "Quels sont les déterminants qui vous incitent à poursuivre la culture du cacao alors que les prix sont au plus bas?", pour plus d'un tiers des producteurs, la plantation reste le seul bien transmissible sûr. S'ils raisonnaient en bons «capitalistes», avec des taux d'intérêt de $60 \%$, ils se moqueraient de ce qui peut arriver à leurs arbres ou à lcur terres dans 5 ou 10 ans, parce que la valeur actuelle de tout «rapport» de cette terre à cet horizon serait de toute façon négligeable, surtout si 
on la multiplie par une probabilité d'éviction assez faible malgré tout.

Plus encore, le maintien des parcelles vivrières depuis l'introduction de la culture de cacao - et quel que soit le prix de la culture de rente - souligne la prééminence d'un système sécuritaire d'autosubsistance. Par ailleurs, pour des prix prévisionnels du cacao de 500 , puis de $700 \mathrm{~F} \mathrm{CFA} / \mathrm{kg}$, les planteurs souhaitent maintenir, voire accroître, leur surface vivrière. Donc, les stratégies vivrières sont aussi des stratégies défensives de "ne pas mettre ses oufs dans le même panier».

Ainsi, les stratégies productives des planteurs sont bien de nature «sécuritaire»: s'assurer au maximum une certaine suffisance alimentaire et s'assurer un revenu minimum grâce au cacao et aux ventes vivrières pour couvrir les dépenses courantes d'exploitation. En effet, plus d'un tiers des planteurs évoquent la sécurité du revenu pour le cacao en 1993. Le cacao retrouve donc un intérêt économique et social, difficilement imaginable selon les valeurs des taux d'intérêt effectivement prises, alors que les prix du cacao sont eux-mêmes loin d'être encourageants.

Tout cela ne veut pas dire que les planteurs sont insensibles aux prix, loin de là. En février 1994, alors que le prix du cacao est fixé officiellement à $300 \mathrm{~F} \mathrm{CFA} / \mathrm{kg}$ - il était à $150 \mathrm{~F} \mathrm{CFA} / \mathrm{kg}$ en début de campagne 1993-1994 - les planteurs ont repris les travaux de nettoyage dans les plantations. Incidemment, cela montre aussi que les prévisions de prix des planteurs pour la prochaine campagne seraient essentiellement les prix «du moment», provenant bien souvent de sources diverses et multiples, glanés auprès des amis, de la famille ou des commerçants fídèles.

Le prix des cultures vivrières, activités alternatives au cacao, est tout aussi important dans les choix des planteurs. Dans certaines régions où la demande est importante et les prix plus attractifs, on voit même se développer des stratégies «agressives» dans le domaine des cultures vivrières. Dans la provin- ce du sud-ouest, le vivrier est devenu une véritable culture de rente, tout autant que le cacao, en raison des potentialités du milieu (sol volcanique) et de la forte demande urbaine (Douala).

Mais cela ne veut pas dire que les planteurs arracheront les pieds de cacao si le prix rechute ou qu'ils se lanceront dans un processus d'intensification pour des prix élevés. En effet, peu de planteurs ont arraché des cacaoyers pour un prix de $150 \mathrm{~F} \mathrm{CFA} / \mathrm{kg}$ en 1993, peu de planteurs ont accru les soins sur les cacaoyères en 1984-1985 alors que les prix atteignaient $450 \mathrm{~F} \mathrm{CFA} / \mathrm{kg}$. Si les effets de la dévaluation sont difficiles à évaluer du fait de l'élasticité limitée des cultures pérennes, les tendances conservatrices semblent toujours dominer. Le vivrier est plus que jamais une culture de sécurité pour couvrir les besoins alimentaires, le cacao constitue toujours une assurance pour la famille. À la question des déterminants de la poursuite de la culture de cacao posée en 1994, ils sont près de $62 \%$ à évoquer le cacao comme une assurance pour la famille.

Ces observations montrent les décisions du maintien de la cacaoculture, comme source sécuritaire de revenu dans une période de fortes instabilités des prix et résultent bien de l'espérance que, sur une période suffisamment longue, le cacao se vende à un bon prix, même si les planteurs sont persuadés qu'à court terme les prix resteront bas et que l'augmentation des prix de 1994-1995 ne se maintiendra pas très longtemps. Encore une fois, c'est assez contradictoire avec la théorie de l'actualisation à taux élevés. Dans un contexte de taux élevé comme au Cameroun, les événements lointains devraient avoir peu d'influence sur les décisions du moment. Ici, l'expérience des crises passées, que l'on pourrait appeler la mémoire collective, explique à bien des égards l'attitude des planteurs.

En outre, l'enquête montre aussi que le risquc et les incertitudes sont dans toutes les dimensions de l'activité et sont très personnelles. Un planteur endetté est contraint de rembourser son prêt avant tout essai de 
replantation; le retour subit d'un frère licencié peut remettre en cause la propriété foncière; on pourrait multiplier les exemples. Ceci explique les difficultés de la prise en compte du risque et des incertitudes à l'aide de théories préétablies. En effet, aux risques classiques de l'offre agricole (perturbations climatiques, dévastation des cultures par des agents pathogènes ou des parasites, variations subites des prix des produits), se juxtaposent des incertitudes d'ordre social (départ subit d'un travailleur, deuil d'un parent entraînant des dépenses imprévues au détriment d'achat d'intrants agricoles) ou psycho- logique (perception plus ou moins optimiste ou pessimiste des tendances de prix). Les premières sont imprévisibles et objectives alors que les secondes appartiennent au registre subjectif et restent soumises aux aléas de l'opinion".

Tout ceci conduit donc à négliger l'actualisation et à supposer que c'est le risque qui conditionne le comportement. Mais peut-on construire un modèle qui nous permet à la fois de vérifier la pertinence des analyses précédentes sur la façon de gérer le risque par le planteur et de valider l'approche théorique du «taux d'actualisation endogène»?

\section{Tentative de construction d'un taux d'actualisation endogène}

\section{Théorisation du comportement des planteurs}

Comme on l'a vu ci-dessus, la programmation multipériodique dynamique s'impose pour représenter le comportement des planteurs, en raison de la durée de vie des cacaoyers. En début d'année t, le ménage est doté de certains facteurs fixes de production (travail, terre, plantations); ses liquidités résultent des performances de son système de production les années passées. A partir de ses anticipations sur les prix futurs et des moyens à sa disposition, on suppose que chaque planteur choisit son assolement afin de maximiser une fonction d'utilité sous des contraintes techniques, financières et de sécurité. La représentation des contraintes techniques et financières est facile, et nous ne nous y attarderons pas ${ }^{5}$. Les contraintes techniques lient le niveau des facteurs techniques

4. Bien évidemment. nous abandonnons ici toute référence à la fameuse distinction entre risque et incertitude. Tout au plus, nous nous référerons à l'idée Bayésienne que l'on peut toujours prendre une probabilité subjective comme mesure de vraisemblance...

5. Elles sont classiques pour ce type de modèle (Boussard, 1971; Leplaideur et al., 1981). On en trouvera le détail chez Alary (1996). employés et les rendements obtenus pour chaque activité et compte tenu des pratiques des années précédentes. En ce qui concerne les contraintes financières, elles traduisent les flux monétaires annuels en fonction de l'épargne et des emprunts des années précédentes.

Il est important, dès à présent, de noter qu'un tel programme peut avoir un horizon de planification infini, même si la maximisation se fait sur un nombre restreint d'années. C'est là l'essence des enseignements du «théorème de l'Autoroute» (Mc Kenzie, 1976). Étant donné un vecteur $\mathrm{x}$ de variables d'état et de décision, qui prend la valeur $x_{t}$ l'année $t$, et qui est soumis à un ensemble de contraintes du type:

$F\left(x_{t}\right)-x_{1-1}<0$, on choisit $\mathrm{v}=\left\{\mathrm{x}_{1}, \ldots, \mathrm{x}_{\mathrm{r}}\right\}$ de manière à maximiser une fonction $U(v)$. On montre alors que sous certaines conditions «classiques» (en particulier, la convexité de F et la «forte» concavité de $U$ ), il existe des dates $t_{1}$ et $t_{2}$ telles que, pour $\mathrm{t}_{1}<\mathrm{t}<\mathrm{t}_{2}$, la valeur optimale $x_{1}{ }^{*}$ de $x_{t}$, à un facteur de proportionnalité près, ne dépend plus ni de la fonction d'utilité $U$, ni de l'horizon de planification $T$, ni du point de départ $x_{0}$, mais seulement de la «technologie»F. Pour $t<t_{1}, x_{t}{ }^{*}$ dépend de $x_{0}$, 
mais ni de U, ni de T. Dès lors, il n'est jamais nécessaire de faire des plans au delà de $t_{1}$, même sur un horizon de planification très lointain. Par ailleurs, les plans pour les années 2 et suivants ne sont pas forcément construits en vue de leur exécution (si de l'information fraîche survient entre temps, on sera conduit à les réviser), mais seulement comme un moyen de traiter l'information concernant l'avenir disponible à l'instant où se prend la décision pour l'année 1, la seule année réellement importante, et qui soit effectivement mise en œuvre (Modigliani, 1952; Modigliani et Cohen, 1951). Tout ceci permet de définir l'horizon de planification comme le temps au-delà duquel il est inutile de faire des plans pour prendre une décision concernant la première période.

Ce théorème, dont il existe des versions en «temps continu», est aussi valable dans un environnement stochastique. Majumdar et Zilcha (1987) démontrent que les propriétés de convergence des modèles de croissance vers une «autoroute» restent valables dans un environnement stochastique, pourvu toutefois que la croissance puisse se poursuivre indéfiniment quelques soient les circonstances. Il devient alors légitime de remplacer les valeurs aléatoires par leur moyenne. En outre, au-delà des perturbations fréquentes au cours du temps, le chemin de croissance se rapproche inéluctablement du chemin optimal en «moyenne».

Ceci suppose toutefois que la croissance ne se soit jamais interrompue et que le décideur puisse à tout moment «repartir». C'est ce qui justifie l'introduction de contraintes de sécurité dans le modèle. Ici, les contraintes de sécurité sont conçues de telles façons que le producteur, dans la plus mauvaise configuration de revenu par unité produite, dispose toujours des moyens nécessaires pour couvrir les dépenses incompressibles d'exploitation et rembourser ses dettes.

Soit $Z_{1}$ le revenu du planteur l'annćc t. Pour qu'il puisse prendre des décisions l'année $t^{\prime}$, il faut cependant qu'il ait réussi à survivre jusque-là. Cela suppose qu'il ait pu obtenir au moins le revenu $Z_{0}$ chaque année entre les années 0 et $t^{\prime}$, où $Z_{0}$ est un revenu minimal annuel qui fixe le seuil de ruine. Soit alors $P_{s}$ (t') la probabilité d'avoir survécu les périodes antérieures à l'année $t$ '. $P_{s}$ est fonction des probabilités $P_{1}$ de survie l'année $t$, soit $P_{t}$ $=\operatorname{Prob}\left(\left\{Z_{4}<Z_{0}\right\}\right) . P_{1}$ est une variable de décision, choisie chaque année par le planteur. On peut donc considérer les $P_{1}$ comme indépendant en probabilité les uns des autres, et on a donc:

$$
P_{s}\left(t^{\prime}\right)=\prod_{t<t^{\prime}} P_{t}
$$

Cependant, $P$, est une variable de décision en quelque sorte indirecte, qui dépend d'autres décisions. Si on admet - ce qui est fort discutable - que le revenu $Z_{4}$ est Gaussien, la probabilité $P_{1}$ ne dépend que de la moyenne et de l'écart type de $\mathrm{Z}_{\mathrm{Z}}$ :

$$
P_{1}=\frac{1}{2} \theta\left(\left[E\left(Z_{t}-Z_{0}\right)\right] / V^{1 / 2}\left(Z_{t}\right)\right)
$$

où $\theta(r)$ est la fonction de répartition de la loi de Gauss au point $r, E(Z)$ est l'espérance mathématique de $Z$, et $V(Z)$ sa variance.

La moyenne $\mathrm{E}(Z$, ) est facile à calculer. $T y$ piquement, on a:

$Z_{t}=\sum_{j} C_{j t} X_{j t}$

où $x_{j}$ l'élément $j$ du vecteur $x_{t}$ défini plus haut, et $c_{j t}$ un coefficient aléatoire - prix ou rendement, ou produit des deux - dont il est raisonnable de supposer la loi de probabilité stationnaire. Alors:

$E\left(Z_{t}\right)=\sum_{j} E\left(c_{j t}\right) x_{i t}$

On remplace chaque coefficient aléatoire par sa moyenne. Il n'en est pas de même de la variance $V\left(z_{1}\right)$. D'abord, les différentes activités d'une année ne sont pas forcément indépendantes en probabilité les unes des autres. On est donc amené à écrire:

$\mathrm{V}\left(\mathrm{Z}_{\mathrm{l}}\right)=\sum_{k} \sum_{l} \omega_{k l} x_{k l} x_{l t}$

où $\omega_{k l}$ représente le coefficient de variance covariance entre $c_{1}$ et $c_{k}$. Cela complique un peu le calcul, en particulier parce que cela suppose que l'on connaisse les $\omega_{k l}$ (on fera 
souvent l'hypothèse qu'ils sont nuls pour $1 \neq k$, ce qui revient à postuler l'indépendance en probabilité des rendements et des prix des différentes cultures, une hypothèse après tout assez raisonnable ${ }^{6}$ ), sans entraîner de difficultés insurmontables. Mais cela ne suffit pas, il convient de se poser des questions sur l'hypothèse de l'indépendance en probabilité du revenu de l'année $t$ et de celui de l'année $\mathrm{t}-1$.

En effet, si le revenu de l'année t-1 a été «mauvais» (bon), il y a beaucoup de chances pour que le planteur ait réduit (augmenté) ses investissements. De ce fait, le revenu de l'année $t$, qui dépend des investissements antérieurs par l'intermédiaire des relations (1) n'est pas indépendant en probabilité des événements qui y surviennent. Dans ces conditions, il faudrait logiquement rajouter dans l'équation (6) un terme exprimant cette covariance.

On peut cependant se demander s'il est nécessaire de recourir à ces complications, compte tenu du cadre de cette étude, et du fait, encore une fois, que le problème n'est pas de développer un programme «optimal», mais de traiter le mieux possible l'information dont on dispose pour l'avenir. Le problème de l'influence des années antérieures sur la variabilité du revenu de l'année $t$ ne se pose que si le sujet anticipe son comportement de ce point de vue, autrement dit, s'il est conscient du fait qu'une mauvaise année

6. En effet, la multiplicité des prix pour les produits vivriers vendus à la porte de la case, sur le marché local ou encore sur le marché urbain, la faiblesse de la demande des planteurs sur ces marchés, amenuisent les relations entre prix des denrées vivrières et prix du cacao. Si les prix des intrants importés (produits chimiques, outillage) fluctuent entièrement en fonction du coût de la monnaie à l'étranger, le prix du cacao dépend quant à lui des coûts de stabilisation fixés par les exportateurs et répond donc à un rapport de force national des intermédiaires dan: la filière. On peut multiplier les exemples en la matière.

L'hypothèse d'indépendance des prix de vente du cacao et des produits vivriers, des prix d'achat sur le marché et du coût de l'emprunt semble la moins mauvaise dans une économie désincarnée où le prix résulte davantage du pouvoir que du marché. peut lui faire ralentir son plan d'investissement, et s'il en tire les conséquences rien n'est moins sûr. C'est pourquoi, afin d'éviter d'avoir à faire intervenir des considérations de ce type dans l'analyse, on a admis ici-de façon encore une fois assez arbitraire - que toute fluctuation de $Z_{\text {l }}$, pour $t<t$ ', est absorbée par des variations du niveau de consommation en $t$, sans effet sur l'épargne, et, par conséquent, qu'il n'y a pas d'influence des aléas des périodes $\mathrm{t}<\mathrm{t}$ 'sur le plan d'investissement jusqu'à $\mathrm{t}^{7}$. Alors la variance de $\mathrm{Z}_{\mathrm{r}}$ dépend seulement des événement en $\mathrm{t}$, et on peut, à partir de (6), calculer la formule (3) assez facilement dans le modèle. Ainsi se trouve-t-on dans les conditions requises pour appliquer Majumbar et Zilcha. Reste à déterminer la fonction d'objectif.

La logique de la théorie néoclassique standard est de maximiser la somme des consommations actualisées sur un horizon infini. C'est le «critère de Faustmann», cher aux forestiers, comme on l'a vu plus haut, soit:

$U=\sum_{t=1}^{\infty} C_{t}(1+i)^{-t}$

où $\mathrm{C}_{\mathrm{i}}$ est la consommation finale de la période $t$, ct $\mathrm{i}$ lc taux d'actualisation. Rien ne s'oppose en principe à calculer l'espérance de ce critère, soit:

$U=\sum_{t=1}^{T} C_{t} P_{S}(t)(1+i)^{-t}$

où $C_{t}$ est la consommation planifiée de la période $t$, et $P_{S}(t)$ la probabilité de survie définie plus haut. Il est remarquable que $P_{S}(t)$ décroît de façon exponentielle avec le temps, comme un facteur d'actualisation. Mais à la différence d'un facteur d'actualisation, il est endogène, et déterminé par le programme.

7. Dans un environnement où les besoins vitaux ont du mal à être satisfaits, il n'est pas abusif de penser que toute augmentation du revenu des producteurs permettra d'améliorer le niveau de consommation des ménages, une partie servira certainement à acheter aussi des produits de luxe dans une société de prestige. 
Enfin, et dans le but de raccourcir l'horizon de planification effectivement utilisé (il aurait été très coûteux en temps de calcul d'avoir un horizon de 50 ans et plus, comme le voudrait logiquement la durée de vie d'une cacaoyère), on a rajouté la valeur résiduelle des arbres au delà de l'horizon, ou plutôt, son espérance, ce qui donne :

$$
U=\sum_{t=1}^{T} C_{t} P_{S}(t)(1+i)^{-t}+P_{S}(T) I_{T}(1+i)^{-T}(7)
$$

où $I_{t}$ représente la somme des valeurs d'actif (arbres plantés, stocks, etc.) et de passif (emprunts non remboursés) l'année t. A nouveau, comme $P_{S}(T)$ tend vers zero quand $T$ augmente indéfiniment, le facteur $P_{S}(T)$ se comporte comme un facteur d'actualisation, et la question qui se pose alors est de savoir quel est le plus important des deux facteurs (1 $+i)^{-t}$ et $P_{S}(t)$ dans l'explication des comportements observés des planteurs.

Pour bien saisir l'enjeu de l'expérience, il importe de considérer que le risque, tel qu'il est pris en compte dans ce modèle, est bien un risque endogène dans le sens où il influence les décisions de consommation à court terme et d'investissement à long terme en fonction des résultats moyens espérés de l'exploitation et donc de sa structure. Il interagit aussi sur le niveau d'endettement, qui est souvent un outil utilisé par les planteurs pour ne pas faire faillite. En effet, lorsque le planteur s'endette, il diminue ses risques de faillite à court terme mais accroît ses risques de faillite à long terme. De fait, la prise de risque pondère le recours à l'emprunt, comme pourrait le faire un taux d'actualisation.

Pour s'assurer de la capacité d'une telle approche du risque à arbitrer les décisions, nous nous proposons de comparer les résultats de simulation avec, puis sans, la prise en compte du taux d'actualisation dans la fonction objectif.

\section{Pertinence du modèle}

Pour essayer de déterminer quels sont en réalité les rôles respectifs du risque et de l'actua- lisation «pure», on a effectué des simulations sur 4 exploitations-types de notre échantillon ${ }^{8}$ : un planteur des classes 1 et 2 [des exploitations «intensives» en travail et en capital du Sud-Ouest comptant respectivement et en moyenne plus de 8000 pieds de cacao et moins de 5000 pieds] et un planteur des classes 3 et 4 [des exploitations «extensives» en capital du Centre et du Sud, comprenant respectivement plus de 3 actifs pour des plantations comptant moins de 5000 cacaoyers et entre 2 et 3 actifs pour des plantations comptant plus de 8000 pieds].

En outre, on suppose que les planteurs ne sont pas en mesure de faire des prévisions à moyen ou long terme: les prix et les coûts unitaires se maintiennent sur toute la période de planification.

Pour ces quatre cas de figure, nous nous proposons de simuler quatre situations:

- Dans la première simulation qui sera la situation «sans actualisation, avec risque», on maximise les consommations non actualisées sur l'horizon de planification, sous la contrainte d'une probabilité de ruine (dont le niveau est endogène).

- Dans la seconde situation, ici nommée de «référence» ou encore situation «sans risque, sans actualisation», on maximise les consommations non actualisées sur l'horizon de planification, mais sans non plus tenir compte du risque, qui est donc ignoré. Il s'agit en somme d'un modèle néoclassique avec taux d'actualisation zéro et sans risque.

- Dans la troisième dite «avec actualisation, sans risque», on maximise, comme il est d'usage courant, la somme des consommations actualisées, avec cette fois, un taux d'actualisation de $60 \%$ (niveau élevé, mais qui correspond aux taux d'intérêt en usage).

- Dans la quatrième, enfin, qui est la situation «avec risque, avec actualisation», c'est la fonction telle qu'elle est décrite en (9): on

8. Les exploitations types sont celles qui représentent le mieux les classes définies à partir de l'analyse des correspondances multiples, suivie d'une classification ascendante hiérarchique sur les données d'enquête de notre échantillon. 
maximise les consommations actualisées sur l'horizon de planification, sous la contrainte d'une probabilité de ruine (dont le niveau est endogène).

Graphique 2. Évolution de la surface cacaoyère pour les différentes situations simulées et pour le planteur représentatif de la classe 1. En ha

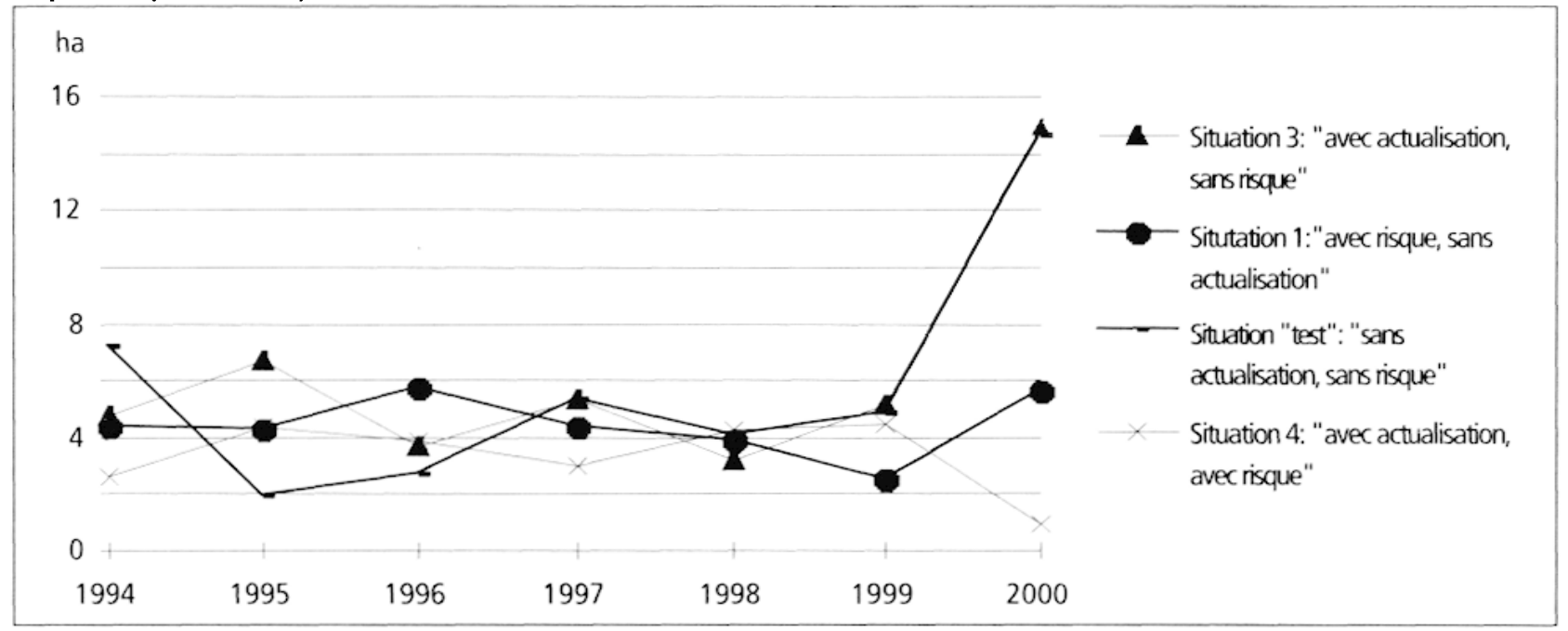

Graphique 3. Évolution de la surface cacaoyère pour les différentes situations simulées et pour le planteur représentatif de la classe 2 . En ha

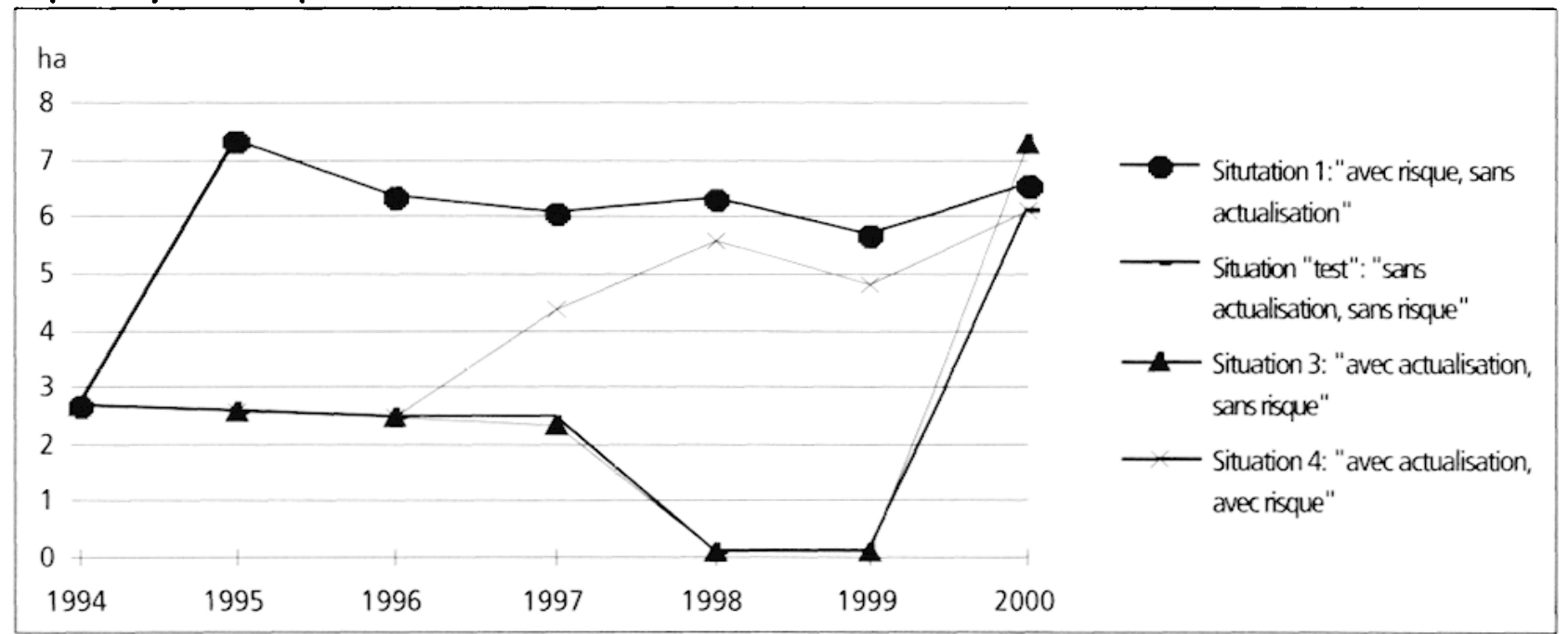

Nous nous intéresserons plus particulièrement à l'évolution des surfaces cacaoyères comme variable illustrative des décisions d'investissement des planteurs à long terme dans la cacaoculture - et à l'épargne comme indicateur des décisions financières. Qu'observe-t-on?

Pour les grandes exploitations dites «intensives» (classe 1), on observe le même phénomène dans les situations «sans risque», où le risque est ignoré dans la fonction objectif (situations 2 et 3 ): le cacao «explose». Même avec un taux d'actualisation très élevé, on est loin de rendre compte du comporte- ment des planteurs, du moins en termes de surfaces, sachant que la majorité des planteurs de cette classe désirent maintenir leurs surfaces plantées. Si on rajoute le risque dans la fonction objectif (situation 4), le modèle conduit à la disparition du cacao. Seule la situation «avec risque et sans actualisation» nous rapproche de la réalité. Les surfaces restent relativement stables sur la période.

Pour les exploitations «intensives» mais de taille moyenne (classe 2 ), bien que les résultats différent, les comportements restent similaires. La situation de "référence» conduit 
toujours à une forte augmentation des surfaces de cacao, mais avec retard. En effet, la situation initiale du planteur représentatif de cette classe est sûrement plus éloignée de l'équilibre que le précédent. Dans la situation 3, «avec actualisation seulement», on observe une spécialisation vivrière, plus rentable à court terme. Or peu de planteurs (moins de $1 \%$ de cette classe) ont osé se lancer dans cette spécialisation. Si on introduit le risque (situations 4 et 1), les surfaces cacaoyères sont maintenues; clles augmentent mêmc.

On constate donc que les planteurs de ces deux classes privilégient leur plantation dans la situation «avec risque», ce qui correspond bien aux intentions de ces planteurs (ils sont plus de la moitié dans chacune des deux classes à vouloir maintenir leur plantation en 1994 suite à la dévaluation du franc CFA). Or, conformément à la théorie classique de l'actualisation et pour un taux d'intérêt élevé, les planteurs auraient tout intérêt à se consacrer aux cultures vivrières. En effet, dans un contexte d'accès difficile à la terre, les cultures vivrières apportent un revenu par unité de surface bien plus attractif. Mais aussi, la survie de l'exploitation n'est plus assurée sur l'horizon.

Pour les exploitations dites «extensives» du Centre (classes 3 et 4 ), non représentées ici, on observe une grande stabilité des surfaces cacaoyères et vivrières quelque soit la situation simulée; ceci reflète bien la faible marge de manoeuvre des petits producteurs. Cependant, il existe des différences notables dans l'arbitrage entre la consommation et l'épargne. Pour le planteur de la «classe 4», seule la prise en compte du risque rend compte du comportement épargnant de la majorité des planteurs de cette classe.

En effet, dans les situations 1 et 4, «avec risque et sans actualisation» ou «avec risque et actualisation", lorsque la probabilité de faillite croit une année donnée, le planteur privilégie son épargne. Si les bénéfices réalisés les années suivantes sont faibles, le planteur préfêre épargner en vue d'accrôitre son capital plantation, qui seul peut agir significativement sur l'utilité dans l'optimisation. Ceci reflète bien le comportement des planteurs dans un contexte très incertain; le planteur préfère investir dans la cacaoculture sorte "d'épargne sur pied"-, que de consommer son surplus dans des activités plus rémunératrices mais aussi plus risquées, comme les cultures vivrières.

Par ce simple raisonnement, on voit comment le risque arbitre les décisions dans le temps en fonction du revenu moyen des exploitants et du niveau du revenu minimum. Il incorpore l'ensemble des paramètres fluctuants dans le temps qui jouent un rôle sur les décisions d'aujourd'hui et de demain de chaque agent. Calculé de façon endogène sur la base des revenus moyens espérés de chaque exploitant et du revenu minimum, il permet donc de mieux cerner ses arbitrages possibles entre le présent et le futur pour éviter une situation de ruine.

\section{Conclusion}

Sur un plan théorique, la confrontation des résultats obtenus par simulation et des résultats d'enquête montre bien que les choix productifs des planteurs et leur façon de gérer les plantations dépendent de leur perception de l'instabilité des prix et de la structure de leur exploitation. Mais ceci a été maintes fois démontré. En revanche, la prise en compte de la notion d'actualisation - comme le produit des probabilités de faillite des années passées- dans la fonction-objectifs ouvre de nouvelles perspectives. Calculé de façon non arbitraire, ce serait en effet un moyen de se rapprocher du véritable taux d'actualisation, qui est - comme on pouvait le pressentir - propre à chaque type d'individu et fluctue en fonction de l'évolution de l'entreprise et de son environnement. Ici, il nous a permis de comprendre d'un point de vue économique la place du cacaoyer comme «assurance pour la famille», alors que tous les indices économiques - tant au ni- 
veau du marché mondial du cacao que du marché national - ne présageaient guère de meilleurs jours pour la cacaoculture.

D'un point de vue pratique, on voit aussi que pour la majorité des planteurs, une diminution du coût du travail salarié, du prix des intrants ou du taux d'emprunt ne modifie guère leur système de production. La stabilisation d'un des coûts à un niveau plus élevé semble même plus favorable dans le sens où elle éloigne les planteurs des situations fortement risquées. Ces ré- sultats de simulation tendent à montrer que l'instabilité du prix de la journée de travail, les difficultés d'approvisionnement régulier en intrants ou les fluctuations des taux d'emprunt constituent les principaux handicaps de cette agriculture de plantation. Donc peut-être, mieux que de s'attaquer au prix, il faudrait améliorer les conditions de marché et de son environnement.

Véronique ALARY • CIRAD EMVT Montpellier. Jean-Marc BOUSSARD • INRA économie, Paris

\section{RÉFÉRENCES BIBLIOGRAPHIQUES}

Alary V. Incertitude et prise de risque en période d'ajustement. Le comportement des producteurs de cacao du Cameroun avant et après 1994. Thèse de doctorat, Paris IPanthéon Sorbonne, 1996, 750 p.

Bateman M.J. Aggregate and Regional Supply Functions for Ghanaian Cocoa, 19461962. In : Measuring Supply and Demand in Underdeveloped Agriculture, Lady Ursula Hicks, editor, Nuffield College, Oxford, 1965, pp. 384-401.

Binswanger H.P. Attitudes Toward Risk: Theoretical Implications of an Experiment in Rural India. In The Économic Journal, 1981, n 91 , pp. 867-890.

Boussard J.-M. Optimal Growth. American journal of agricultural economics, 1971.

Boussard J.-M. Variations sur le critère de Faustmann. 1995, version provisoire, 8 p.

Courade G., Grangeret I., Janin P. La vente des joyaux du Prince, la redistribution du gâteau national ou la disparition de l'agriculture de plantation? Les enjeux de la libéralisation des filières café-cacao au Cameroun. In Politique africaine, éd. Karthala, 1991, n 44, pp. $121-128$.

Courade G., Alary V. Les planteurs camerounais ont-ils été réévalués? In Politique africaine, éd. Karthala, 1994, ${ }^{\circ}$ 54, pp. 74-87.
Faustmann M. Berechnung des Wertes Waldboden sovie noch nicht handare Holzbestände für die Waldwirtchaft besitzen. Allgemeine Forst und Jagd Zeitung 25, 1849, pp. 441-455.

Leplaideur A., Longuepierre G., Waguela A Modèle «3C»: Cameroun-Centre-Sud-Cacaoculture. IRAT, Montpellier-Paris, 1981, $236 \mathrm{p}$.

Majumdar M., Zilcha $\mathrm{L}$ Optimal Growth in a Stochastic Environment: Some Sensitivity and Turnpike Results. Journal of Economic Theory 43, 1987, pp. 116-133.

Modigliani F. Production Planning Over Time, and the Nature of the Expectation and Planning Horizon. Econometrica 20 (3), 1952, pp. 481-482.

Modigliani F., Cohen K..J. The role of Anticipations and Plans in Economic Behavior, and their Use in Economic Analysis and Forecasting. Mimeo, University of Illinois, Urbana, 1951.

Mc Kenzie L. Turnpike Theory. Econometrica, 1976, 44(s), pp. 841-865.

Samuelson P.A. Economics of Forestry in an Evolving Society. Journal of Forest Economics, 1995, vol. $1, \mathrm{n}^{\circ} 2$.

Terreau J.-P. Principe de gestion des investissements en forêt. Thèse de doctorat, Université de Toulouse I, 1990. 